\title{
Therapie mit oder ohne Pleurodese?
}

\author{
Nach einem ersten Spontanpneumothorax ist die Rezidivrate \\ hoch, insbesondere bei Jungen und Männern im Alter von \\ 15-40 Jahren: 16 bis $52 \%$ der Patienten erkranken erneut. \\ J.-S.Chen et al. haben in ihrer Studie die Rezidivraten nach \\ einer Standardtherapie und der zusätzlichen Pleurodese mit \\ Minocyclin verglichen.
}

Lancet 2013; 381: 1277-1282

Insgesamt 214 Patienten nahmen an der randomisierten, offenen Parallelgruppenstudie teil. Sie waren zwischen 15 und 40 Jahre alt und hatten einen ersten Spontanpneumothorax mit einem Luftspalt $>2 \mathrm{~cm}$ und anschließend kompletter Lungenentfaltung im Röntgenbild. In allen Fällen erfolgte die Aspiration und Drainage über einen Pigtail-Katheter. Die Patienten nahmen Paracetamol zur Schmerzprophylaxe ein und erhielten auf Wunsch zusätzlich Pethidin. Bei 106 Erkrankten wurden zudem $300 \mathrm{mg}$ Minocyclin in den Pleuraspalt instilliert. Der primäre Studienendpunkt war die Rückfallhäufigkeit innerhalb eines Jahres.

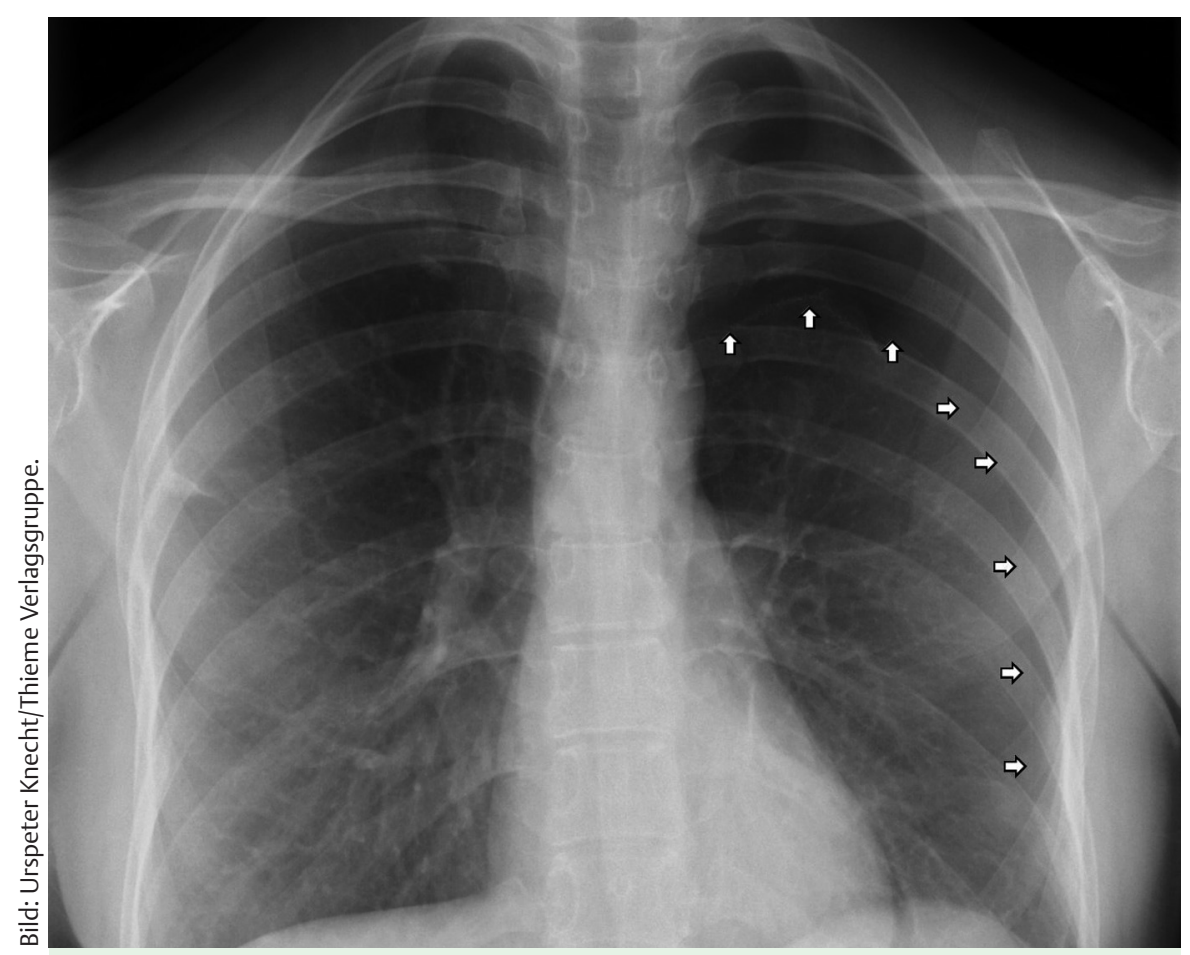

Eine Minocyclin-Pleurodese sollte als Ergänzung zur Standardtherapie mit Aspiration und Drainage bei einem Spontanpneumothorax erfolgen, so die Autoren. Im Bild: Pneumothorax p.a.

griffe (29,2 vs. 43,5\%; $p=0,034)$. Lockere, leicht lösbare Adhäsionen waren nach der Minocyclin-Gabe häufiger. Sie beeinträchtigten den Erfolg thorakoskopischer Behandlungen nicht.

Die medikamentöse Pleurodese war schmerzhafter, obwohl vor dem Eingriff eine intrapleurale Lidocain-Anästhesie erfolgt war. Der Bedarf (67,9 vs. 19,4\%) und die Gesamtdosis von Pethidin waren in der Pleurodese-Gruppe deutlicht höher. Dies galt nur in der Anfangsphase. Nach der Entfernung des Pigtail-Katheters traten in der Kontrollgruppe öfter Schmerzen auf. 6 Monate nach der Behandlung traten in beiden Gruppen kaum Beschwerden auf.

\section{Fazit \\ Die Minocyclin-Pleurodese war sicher und effizient. Die Rezidivrate von ersten Spontanpneumothoraces wurde deut- lich gesenkt. Die Autoren empfehlen die Minocyclin-Pleurodese als Ergänzung zur Standardtherapie mit Aspiration und Drainage.}

\section{Dr. Susanne Krome, Melle}

\section{Kommentar zur Studie}

P. Lee sieht die Ergebnisse der Studie kritisch. Entscheidend sei, dass keine prätherapeutische Stratifizierung der Patienten erfolgt ist. Emphysem-ähnliche Veränderungen und eine verstärkte Porosität der Pleura seien häufige pathophysiologische Ursachen und nicht abgeklärt worden. Wenn ein Patient Lungenveränderungen aufweise, sollte ein chirurgischer Eingriff bereits vor einem Rezidiv diskutiert werden. Die kurze Beobachtungszeit erlaube zudem keine Aussage über die spätere Operationsnotwendigkeit. Die primären Erfolgsraten von Minocyclin (92,5\%) und Talkum (91\%) seien vergleichbar, so Lee. Lancet 2013; 381: 1252-1254

\section{Checklisten $\sqrt{ }$}

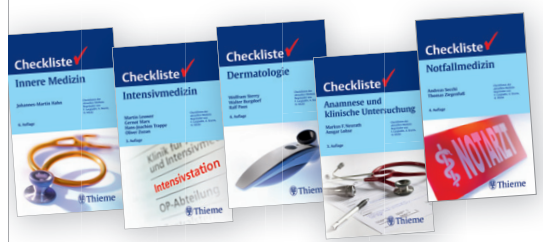

Einfach auf thieme.de bestellen. 